\title{
ADAPTIVE TWO-PHASE PARTICLE FINITE ELEMENT MODEL FOR SOFT SOIL EXCAVATIONS IN PARTIALLY SATURATED SOILS
}

\author{
ABDIEL R. LEON AND GÜNTHER MESCHKE \\ Ruhr University Bochum \\ Institute for Structural Mechanics \\ Universitätsstraße 150, 44801, Bochum, Germany \\ e-mail: guenther.meschke@rub.de
}

Key words: Cutting tool-soil interaction, partially saturated soil, two-phase Particle Finite Element model, hypoplastic soil model, soil cutting excavation experiments

\begin{abstract}
A coupled solid velocity-water pressure (vp) Particle Finite Element (PFEM) formulation in conjunction with a hypoplastic model for sandy soils is presented. Large deformations of the soil and the ever-evolving topology of the ground free surface, key features characterizing the cutting tool-soil interaction, are efficiently captured via the PFEM. The utilization of a hypoplastic constitutive formulation allows for the realistic modeling of the non-linear behavior of the soil deformable skeleton. The hydromechanical description of the partially saturated soft soil is completed by incorporating the van Genuchten hydraulic model for the calculation of the Soil Water Retention Curve (SWRC) in combination with the Karman-Cozeni equation for the estimation of the soil permeability. Finally, computational simulations of tool-soil interaction performed in sand are compared against experiments and the capabilities of the proposed model are demonstrated.
\end{abstract}

\section{INTRODUCTION}

Tool-soil interactions in water-saturated soft soils are relevant in a variety of engineering activities such as agricultural tillage, submarine dredging and mechanized tunneling for underground constructions. The efficiency of these operations are largely dependent on the torque and energy requirements of the cutting tools and the tool wear during the excavation of the ground. Computational methods open possibilities for a detailed investigation of different aspects connected with the hydromechanical behavior of fully or partially saturated soil during the tool-soil interaction, e.g. ground deformations, effective stresses and pore pressure distributions in the ground. Additionally, reaction forces generated on the cutting tool in dependence of the soil properties and operational parameters 
of the excavation procedure are easily obtainable. This information can be subsequently utilized in the design of more efficient and durable cutting tools with optimal geometry and configuration which may result in substantial improvements in the excavation operation as a whole.

In this contribution, a two-phase Particle Finite Element (PFEM) [1] numerical model suitable for the analysis of tool-soil interactions in partially saturated soils is presented. The tool-soil interactions are characterized by very large soil deformations, continuously changing spatial boundaries and the development of localized shear deformations in the ground, which are properly handled by the PFEM. The non-linear mechanical response of the soil skeleton is modeled by means of a hypoplastic formulation for granular materials. The hydraulic behavior of the partially saturated soil mixture is described by appropriate constitutive relations characterizing the degree of saturation and the saturated hydraulic conductivity of the soil. The capabilities and limitations of the proposed hypoplastic twophase PFEM model are demonstrated by means of computational re-analyses of laboratory tests of tool-soil interaction performed with an in-house excavation device in initially fully saturated sand. Computed and measured results pertaining to the topology of the excavated ground and the reaction forces on the cutting tool are compared and discussed.

2 NUMERICAL MODEL

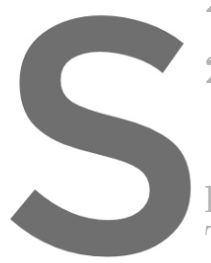

2.1 Velocity-pressure Particle Finite
A coupled two-phase PFEM model, bas
partially saturated soils proposed in [2] is deve

The balance of momen

format, reads

Register for free at https//www.scipedia.cठmato downloadnthe version without the wattermark

where $\dot{v}_{s}$ is the material time derivative of the soil skeleton velocity, $\nabla \cdot \sigma$ the divergence of the total Cauchy stress tensor, $\nabla=\frac{\partial()}{\partial \boldsymbol{x}}$ the spatial gradient operator, $\boldsymbol{x}$ the vector of spatial coordinates, $t$ the time, $\rho \boldsymbol{b}$ the vector of body force, $\rho=(1-n) \rho_{s}+n \rho_{w}$ is the averaged density of the mixture, $\rho_{s}, \rho_{w}$ the intrinsic densities of the solid and water constituents, respectively, and $n$ the porosity of the medium.

In this work, the two-phase PFEM model is coupled to a hypoplastic constitutive formulation which also incorporates the void ratio of the soil as state variable. Therefore, the porosity is computed in terms of the void ratio $e$ via the expression

$$
n=\frac{e}{1+e},
$$

where $e$ is computed by means of the following evolution equation

$$
\dot{e}=(1+e) \operatorname{tr}(\boldsymbol{d}) \text {. }
$$


In Eq. (3), $\boldsymbol{d}=\frac{1}{2}\left(\nabla \boldsymbol{v}_{s}+\nabla \boldsymbol{v}_{s}^{T}\right)$ is the rate of deformation tensor.

The mass balance equation for the fluid phase is given as

$$
n \frac{\partial S_{w}}{\partial t}+\nabla \cdot \hat{\boldsymbol{v}}_{w s}+S_{w} \nabla \cdot \boldsymbol{v}_{s}=0 . \quad \text { in } \Omega
$$

where $S_{w}$ is the degree of water saturation of the soil mixture and $\hat{\boldsymbol{v}}_{w s}$ the averaged relative flow velocity, computed with the help of Darcy's law as

$$
\hat{\boldsymbol{v}}_{w s}=-\frac{k_{w}}{\rho_{w} g}\left(\nabla p_{w}-\rho_{w} \boldsymbol{g}\right)
$$

with $k_{w}$ as the hydraulic conductivity of the water phase (given in $\mathrm{m} / \mathrm{s}$ ) and $g=\|\boldsymbol{g}\|$.

The governing equations (1) and (4) are supplemented by the following boundary conditions

$$
\begin{array}{rrr}
v_{s}-\hat{v}_{s}=0, & \text { on } \Gamma_{v}, \\
\sigma \cdot n-\hat{t}=0, & \text { on } \Gamma_{\sigma}, \\
p_{w}-\hat{p}_{w}=0, & \text { on } \Gamma_{p},
\end{array}
$$
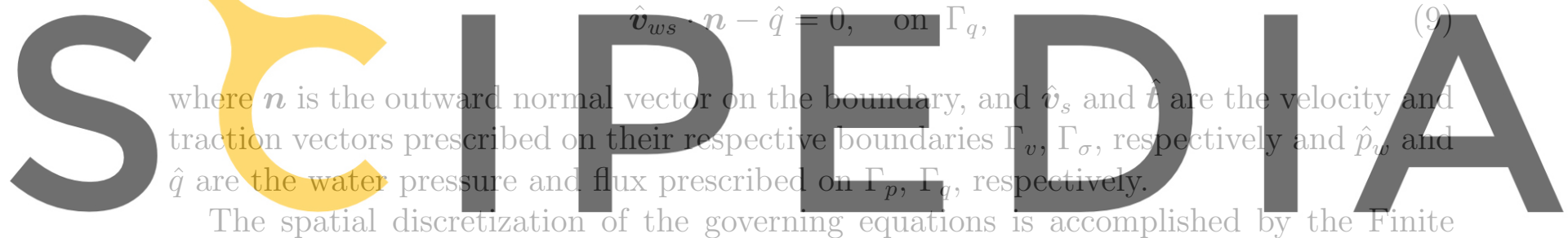

The spatial discretization of the governing equations is accomplished by the Finite Register for free at hthod. Test functions and the primary field variables, of the formulation are

$$
\begin{aligned}
& \boldsymbol{v}_{s} \approx \sum_{I=1}^{N N} N_{I} \overline{\boldsymbol{v}}_{s I}, p_{w} \approx \sum_{I=1}^{N N} N_{I} \bar{p}_{w I}, \\
& \delta \boldsymbol{v}_{s} \approx \sum_{I=1}^{N N} N_{I} \delta \overline{\boldsymbol{v}}_{s I}, \quad \delta p_{w} \approx \sum_{I=1}^{N N} N_{I} \delta \bar{p}_{w I},
\end{aligned}
$$

where $N_{I}$ are linear shape functions associated with node $I$ and $N N$ is the number of nodes per element. In this work, 3-node linear triangles (containing one Gauss point per element) are used for 2D analyses.

The semi-discrete element residuals obtained from Eqs. (1) and (4) are integrated over 
the current element domain $\Omega_{n}^{e}$ and written in matrix notation as

$$
\begin{aligned}
& { }_{e} \boldsymbol{R}_{v}^{n+1}=\int_{\Omega_{e}^{n}} \boldsymbol{N}_{v}^{T} \rho \boldsymbol{N}_{v} d \Omega_{e}^{n} \dot{\overline{\boldsymbol{v}}}_{s}+\int_{\Omega_{e}^{n}} \boldsymbol{B}^{T} \boldsymbol{\sigma}^{\prime} d \Omega_{e}^{n}-\int_{\Omega_{e}^{n}} \boldsymbol{B}^{T} S_{w} \mathbf{1} \boldsymbol{N} d \Omega_{n}^{e} \overline{\boldsymbol{p}}_{w} \\
& -\int_{\Omega_{n}^{e}} \boldsymbol{N}_{v}^{T} \rho \boldsymbol{b} d \Omega_{e}^{n}-\int_{\Gamma_{e}^{n}} \boldsymbol{N}_{v}^{T} \hat{\boldsymbol{t}} d \Gamma_{e}^{n}=\mathbf{0}, \\
& { }_{e} \boldsymbol{R}_{p}^{n+1}=-\int_{\Omega_{e}^{n}} \boldsymbol{N}^{T} n \frac{\partial S_{w}}{\partial p_{c}} \boldsymbol{N} d \Omega_{e}^{n} \dot{\overline{\boldsymbol{p}}}_{w}+\int_{\Omega_{e}^{n}} \boldsymbol{N}^{T} S_{w} \mathbf{1}^{T} \boldsymbol{B} d \Omega_{e}^{n} \overline{\boldsymbol{v}}_{s}-\int_{\Omega_{e}^{n}} \nabla \boldsymbol{N}^{T} \hat{\boldsymbol{v}}_{w s} d \Omega_{e}^{n}, \\
& +\int_{\Gamma_{e}^{n}} \boldsymbol{N}^{T} \hat{q} d \Gamma_{e}^{n}+{ }_{e} \boldsymbol{f}_{\mathrm{stab}}^{n+1}=\mathbf{0}
\end{aligned}
$$

where $N_{v}$ and $N$ are matrices containing the shape functions, $\boldsymbol{B}$ denotes the straindisplacement matrix computed in the current configuration, $\sigma^{\prime}$ is the Cauchy effective stress, $p_{c}=-p_{w}$ the capillary pressure and $e_{e} \boldsymbol{f}_{\text {stab }}^{n+1}$ the element stabilization vector defined in this work based on the Fluid Pressure Laplacian (FPL) stabilization procedure for partially saturated media, proposed in [3].

The temporal discretization of the semi-discrete balance of momentum equation is performed via the $\alpha$-Bossak integration scheme [4]. The rate of the water pressure $\dot{p}_{w}$ in the mass balance equation is approximated
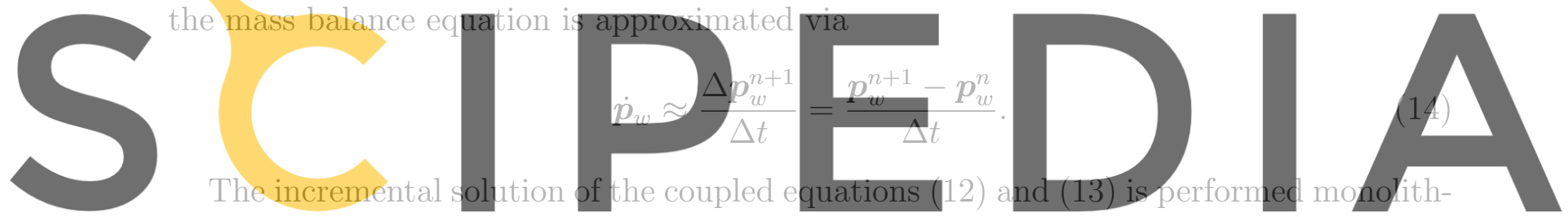

ically by means of an Newton-Rapshon procedure. The nodal increments of the velocities

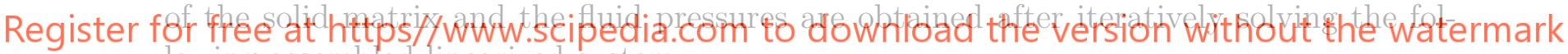
lowing assembled linearized system

$$
[\boldsymbol{K}]_{n+1}^{i}\left[\begin{array}{c}
\Delta \overline{\boldsymbol{v}}_{s} \\
\Delta \overline{\boldsymbol{p}}_{w}
\end{array}\right]_{n+1}^{i+1}=-\left[\begin{array}{l}
\boldsymbol{R}_{v} \\
\boldsymbol{R}_{p}
\end{array}\right]_{n+1}^{i}
$$

with $i$ as the current iteration number, $\boldsymbol{K}^{n+1}$ the system tangent matrix and $\boldsymbol{R}_{v}^{n+1}, \boldsymbol{R}_{p}^{n+1}$ the assembled residual vectors of Eqs. (12) and (13).

Equation (15) has been numerically solved with an iterative Biconjugate Gradient Solver (BiCGS) in combination with an Incomplete LU factorization (ILU) preconditioner.

\subsection{The Particle Finite Element method}

The PFEM [1] is an Updated Lagrangian global re-meshing procedure suitable for the robust numerical analysis of solid and fluid problems characterized by large deformation and strains, using a FEM-based solution approach. In particular, the Lagrangean nature of the method provides the PFEM with superior capabilities in the treatment of evolving spatial boundaries and substantial changes in the topology of the spatial domain. In 
this method, the spatial discretization of the problem is accomplished by means of nodes (denoted as particles) which are allowed to move freely according to the solution of the governing equations. These equations are solved in a time-staggered fashion using standard FEM procedures with the help of a computational mesh. After each solution step, the elements comprising the mesh are cleared and the information of kinematic and state variables is transferred to the nodes.

In standard versions of the PFEM, the finite elements are generated by means of the Extended Delaunay Tessellation (EDT) technique [5]. The EDT is a mesh triangulation procedure that enforces the generation of well-shaped elements with optimal aspect ratios and maximal internal angles. After construction of the mesh, domain boundaries are detected by means of the $\alpha$-shape technique [5], where elements are preserved or discarded on the basis of a geometric criterion involving their characteristic length. This procedure is performed at each time step, thus allowing to efficiently capture complex boundaries and evolving free surfaces.

During the excavation process of highly compacted soils, localized deformations of the ground in the form of shear bands, may propagate from the cutting tool towards the free surface. To enhance the capabilities of the PFEM concerning the representation of these shear bands, a local adaptive mesh refinement strategy is implemented. In this approach,

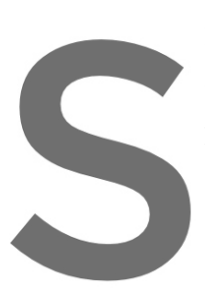
nodes are inserted

designed to track the relative increase of the
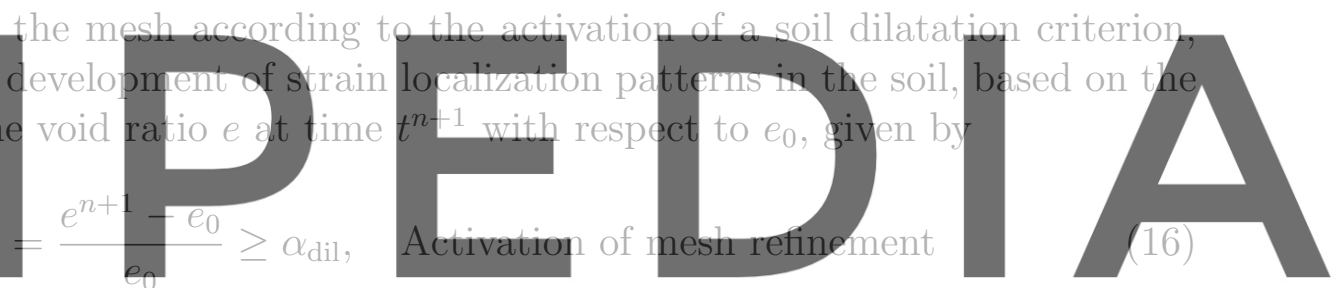

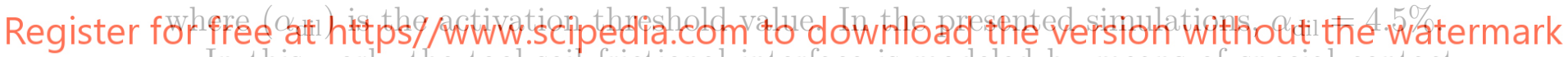

In this work, the tool-soil frictional interface is modeled by means of special contact elements using the Continuum Constraint Method (CCM) [6]. In the CCM, the elements discretizing the contact interface are of the same kind and order of those comprising the main domain (i.e. linear triangular elements in 2D) and the contact behavior is reflected only at the constitutive level. In this sense, the response of contact elements is modeled using a different material model and properties different from those employed for the discretization of the domain.

\subsection{Constitutive relations}

The determination of the degree of water saturation $S_{w}$ of the soil mixture is accomplished by means of a Soil-Water Characteristic Curve (SWCC) according to the van Genuchten model [7]. The SWCC relates $S_{w}$ to the matrix suction $h_{\text {suc }}$ (utilized herein in $\mathrm{cm}$ )

$$
S_{w}=S_{w}^{r}+\frac{S_{w}^{s}-S_{w}^{r}}{\left(1+\left(\alpha_{v g} h_{\mathrm{suc}}\right)^{n}\right)^{m}},
$$


where $S_{w}^{r}$ and $S_{w}^{s}$ denote, the residual and maximum saturation degrees, respectively, $\alpha_{v g}$ and $n$ are independent parameters to be calibrated from hydraulic experimental data of the soil and $m=1-\frac{1}{n}$.

The hydraulic conductivity of the partially saturated porous medium $k_{w}=k_{w}^{r} k_{w}^{\text {sat }}$ is obtained from the scaling of its saturated value $k_{w}^{\text {sat }}$ by the relative permeability coefficient $k_{w}^{r}$. The saturated hydraulic conductivity is estimated in accordance to the KozeniCarman permeability model [8], given here in terms of the void ratio $e$,

$$
k_{w}^{\mathrm{sat}}=C \frac{g}{\rho_{w} \mu_{w}} \frac{e^{3}}{S^{2} G_{s}^{2}(1+e)},
$$

where $C$ is the shape factor of the particles, $G_{s}=\frac{\rho_{s}}{\rho_{w}}$ is the specific gravity of the soil grains and $\mu_{w}$ the dynamic viscosity of the water $\left(\mu_{w}=1 \times 10^{-3} \mathrm{~Pa} \cdot \mathrm{s}\right.$ at $\left.20^{\circ} \mathrm{C}\right)$.

The relative hydraulic conductivity, on the other hand, is computed in terms of the effective degree of saturation $S_{w}^{e}$ as

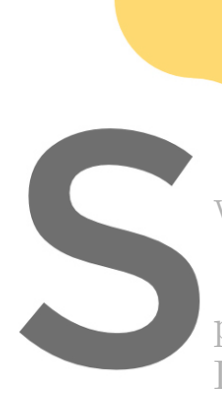

$k_{w}^{r}=\sqrt{S_{w}^{e}}\left[1-\left(1-\left(S_{w}^{e}\right)^{\frac{1}{m}}\right)^{m}\right]^{2}$
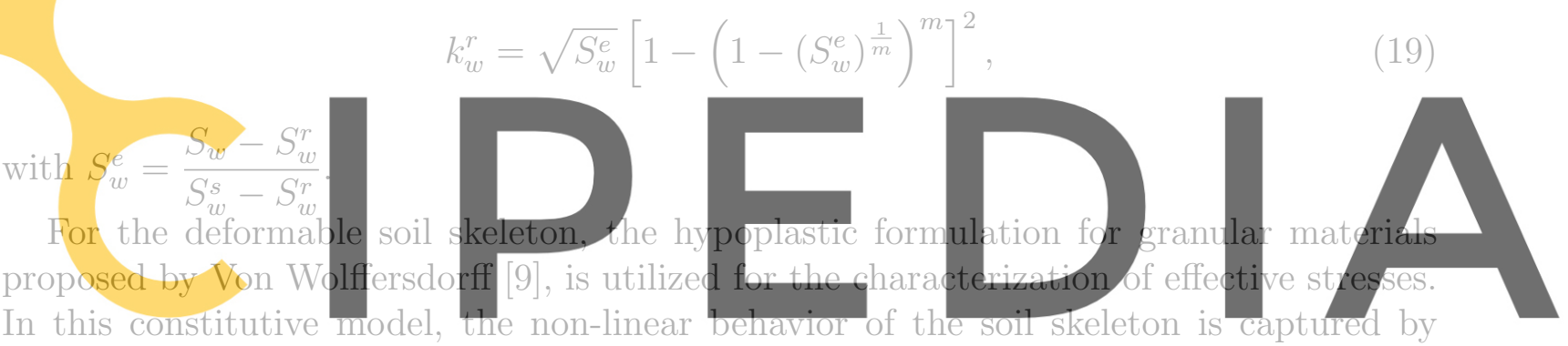

means of the following tensorial rate form

Register for free at https//wWw.scipedia.com to download the version without the watermark

$$
\stackrel{\circ}{\sigma}^{\prime}=f_{b} f_{e}\left(\boldsymbol{L}: \boldsymbol{d}+f_{d} \boldsymbol{N}\|\boldsymbol{d}\|\right) \text {, }
$$

where $\boldsymbol{L}$ and $\boldsymbol{N}$ are fourth-order and second-order constitutive tensors, respectively, and $f_{b}$ and $f_{e}$ are scalar functions utilized to account for the non-linear dependence of the soil stiffness on the mean skeleton pressure and the soil density, respectively. The factor $f_{d}$ is utilized to reproduce the volumetric dilatancy observed in dense specimens.

\section{COMPUTATIONAL SIMULATION OF EXCAVATION OF SANDY SOIL}

\subsection{Experimental setup and material parameters}

An excavation testing device [10] has been designed and constructed at Ruhr University Bochum for the investigation of tool-soil interaction processes in both dry and partially saturated soft soils. It consists of a rectangular soil container (width $23 \mathrm{~cm}$, length 140 $\mathrm{cm}$, height $53 \mathrm{~cm}$ ) enclosed by transparent plastics (Plexiglass) (see Fig. 1). As excavation tool, a rectangular blade made of Plexiglas with a height of $25 \mathrm{~cm}$ is employed. The motion of the blade is controlled by a trolley driven by a stepper motor and supported on dual 
rail-linear bearing systems. Additionally, four force transducers mounted on the cutting tool allow for the measurement of the generated reaction forces during the excavation process.

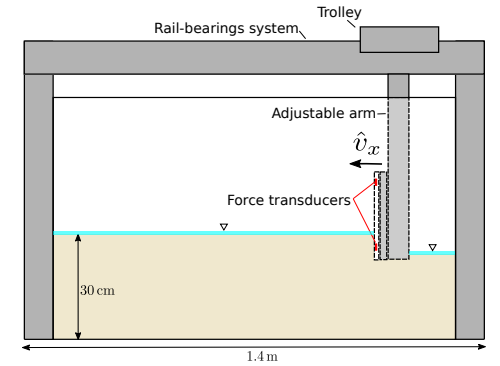

(a) Experimental setup

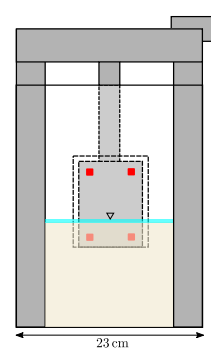

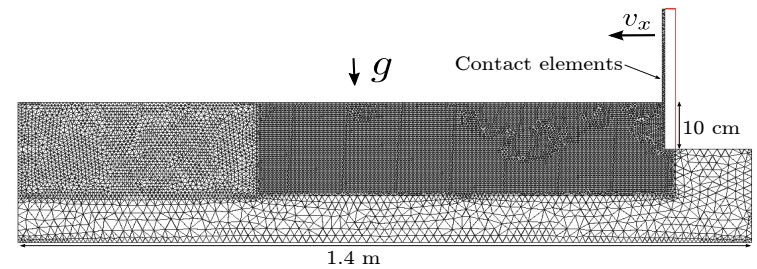

(b) Numerical model

Figure 1: Experimental and numerical analysis of tool-soil interaction in soft soils: a) Schematic illustration of the excavation device; b) PFEM model with geometry and boundary eonditions.

The experiments are characterized by horizontally displacing the cutting tool along the

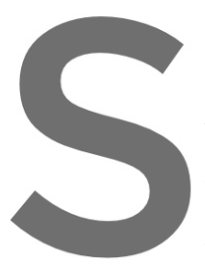
length of the soil bin. mately $450 \mathrm{~mm}$. The advance the penetration depth experiment is approxin gently pouring water ground surface.
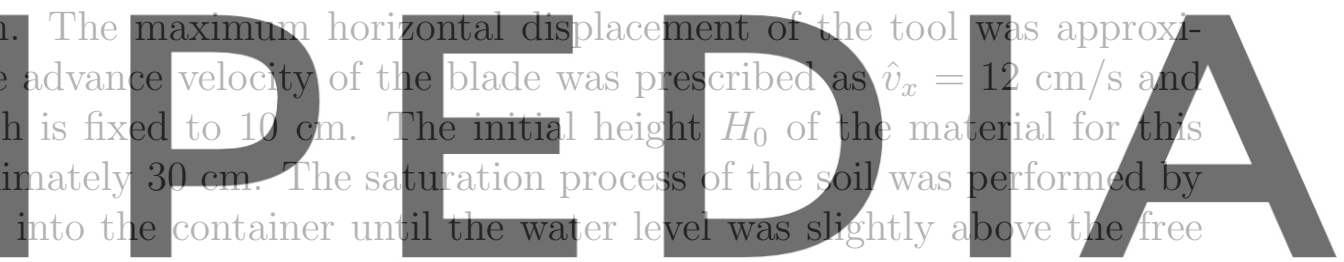

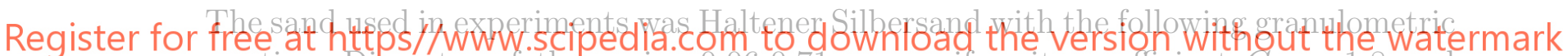
properties: Diameter of the grains $0.06-0.71 \mathrm{~mm}$, uniformity coefficient $\mathrm{C}_{u}=1.8$ and $d_{50}=0.23 \mathrm{~mm}$. The measured friction angle at repose is $33^{\circ}$. The tool-soil interface friction angle is $\delta=13^{\circ}$ [10] for dry sand and has been increased to $\delta=18^{\circ}$ accounting for the observed increase of the sliding friction coefficient with very high water content in the soil, pointed out in [11]. The minimum and maximum void ratios (at zero mean skeleton pressure) for this sand are $e_{d 0}=0.557$ and $e_{i 0}=0.901$, respectively. The critical void ratio (at zero mean skeleton pressure) is set to $e_{c 0}=0.90$. The sand is assumed to be in a dense state with an initial void ratio $e_{0}=0.66$ (relative density $I_{d}=0.70$ ).

In this work, the hypoplastic parameters for Karlsruhe fine sand (diameter of the grains: 0.06-0.3 $\mathrm{mm}, C_{u}=1.5$ and $d_{50}=0.14 \mathrm{~mm}$ ) [12] are adopted due to insufficient laboratory data of Silbersand for a proper calibration procedure. These parameters are collected below in Table 1.

It should be noted that, since the soil excavations herein presented are performed at ground level, where effective stresses are comparatively low, we have reduced the granular hardness $h_{s}$ from its original value i.e. $4 \times 10^{9} \mathrm{~Pa}$ (calibrated for mean skeleton pressures above $20 \mathrm{kPa}$ ), to $2.25 \times 10^{7} \mathrm{~Pa}$, as suggested in [13], to mitigate overestimations of ground 
Abdiel R. Leon, and Günther Meschke

Table 1: Hypoplastic parameters utilized for Silbersand. In parentheses $(\cdot)$, the value of $h_{s}$ calibrated for a mean skeleton pressure of $p^{\prime}>20 \mathrm{kPa}$ is included.

\begin{tabular}{cccccccc}
\hline$\varphi_{c}\left[^{\circ}\right]$ & $h_{s}[\mathrm{~Pa}]$ & $\alpha_{H}$ & $n$ & $\beta_{H}$ & $e_{i 0}$ & $e_{c 0}$ & $e_{d 0}$ \\
\hline 33 & $2.25 \times 10^{7}\left(4 \times 10^{9}\right)$ & 0.14 & 0.27 & 2.5 & 0.901 & 0.9 & 0.557 \\
\hline
\end{tabular}

heaving predicted by the hypoplastic model [13]. The SWCC for Silbersand is generated using the parameters displayed in Table 2 , and the saturated hydraulic conductivity $k_{w}$ has been estimated using Eq. (18).

Table 2: Parameters for the SWCC of Silbersand according to the van Genuchten's model.

\begin{tabular}{cccccc}
\hline$e_{0}$ & $S_{s}$ & $S_{r}$ & $\alpha_{v g}$ & $m$ & $n$ \\
\hline 0.66 & 1 & 0.06 & 0.02913 & 0.7616 & 4.1942 \\
\hline
\end{tabular}
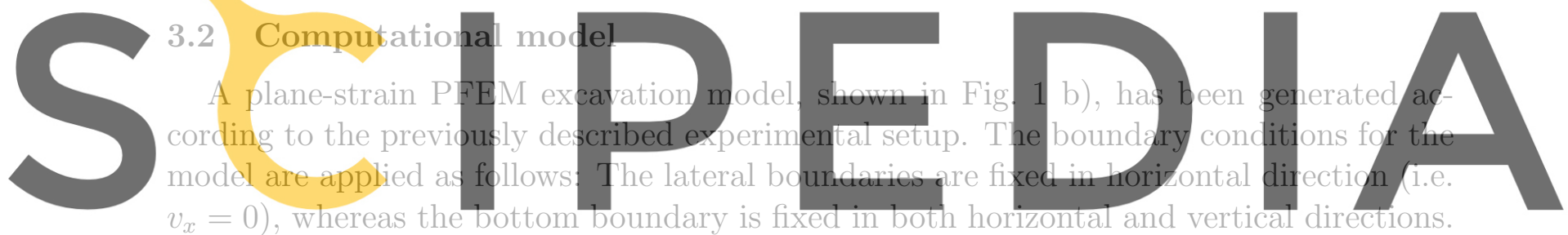

A prescribed velocity of $\hat{v}_{x_{0}}=1.2 \mathrm{~mm} / \mathrm{s}$ is imposed on the rigid cutting tool. At the

Register for free at https/bww.N. scipedia. Gom to download the version without the watermark

bottom boundaries are assumed as impervious. The PFEM mesh consists of 19500 linear triangular elements with sizes ranging from $5.5 \mathrm{~mm}$ near the cutting tool to $18.5 \mathrm{~mm}$ in the right boundary. At the contact interface the elements size is $5 \mathrm{~mm}$ The hypoplastic model is initialized with effective stresses $\sigma_{y 0}^{\prime}=\gamma^{\prime} z$ and $\sigma_{x 0}^{\prime}=K_{0} \sigma_{y 0}^{\prime}$, where $\mathrm{z}$ is the depth measured from the free surface and $\gamma^{\prime}$ denotes the effective unit weight of the soil sample. The lateral earth pressure coefficient $K_{0}=0.46$ is calculated according to Jaky's formula $[14]$.

\subsection{Computed soil deformations and reaction forces on the cutting tool}

Figure 2 presents snapshots of the deformed soil for a tool displacement of $S_{x}=300$ $\mathrm{mm}$ as obtained from the experiment and the numerical simulations using the proposed adaptive PFEM model for fully and partially saturated soft soil. Laboratory results show a staggered topology of the ground free surface. This is produced by the progressive activation of shear bands during the soil cutting process. Numerical predictions concerning the topology of the excavation front qualitatively agree with experiments. In particular, 
the two-phase PFEM model is able to capture to a good extent the observed shear bands. Within these shear bands, the soil undergoes dilatation which is then utilized by the adaptive refinement scheme for the insertion of nodes and creation of new elements, as seen in Fig. 2 b). Details are contained in [15].

The height of the excavation pile ahead of the tool obtained in the test and the PFEM analysis are compared. The maximum height in the laboratory test, measured from the base of the tool, is approximately $197.5 \mathrm{~mm}$, whereas the length of the pile, considered from tool-soil interface, is $\approx 404 \mathrm{~mm}$. According to the PFEM model, the maximum height of the pile is $206 \mathrm{~mm}$ whereas its length is $345 \mathrm{~mm}$. These results yield relative errors of $4.3 \%$ and $14.6 \%$ for the maximum height and length of the excavation heap, respectively.

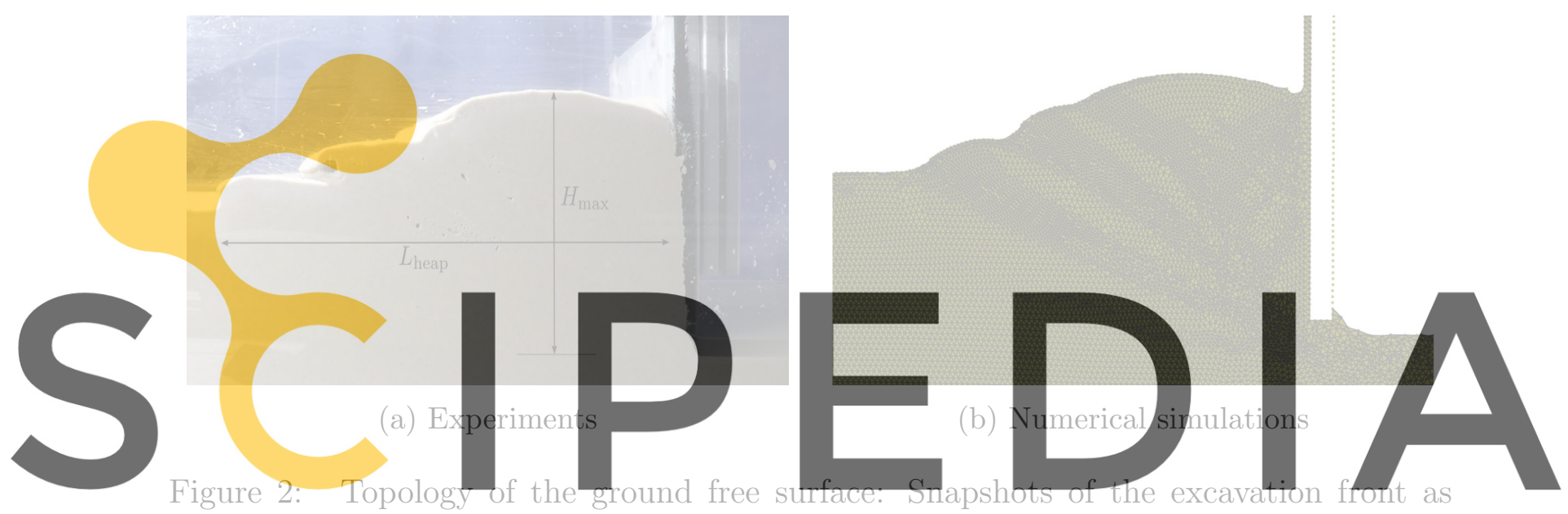

obtained from a) experiments and b) numerical simulations for a tool horizontal displace-

Register for free at httos/ hwwwescipediag.Com to download the version without the watermark

Figure 3 a) illustrates the computed and measured evolutions of tool reaction forces as a function of the horizontal tool displacement, considering the reference simulation case (i.e. using $\varphi_{c}=33^{\circ}$ ). Measurements from five different experimental runs are displayed in light blue lines. The force-displacement plot indicates, that the reaction forces are increasing according to an oscillatory pattern associated with the sequential activation of shear bands in the soil. In the shear bands, the material locally shows strong dilatation, and intense negative pore pressures develop. The computed reaction forces lie within experimental range, however, the proposed numerical model fails to reproduce the aforementioned oscillatory pattern. This issue might be connected to inherent limitations of the utilized hypoplastic model to fully capture the localized pore pressure variations within the shear deformation bands. 


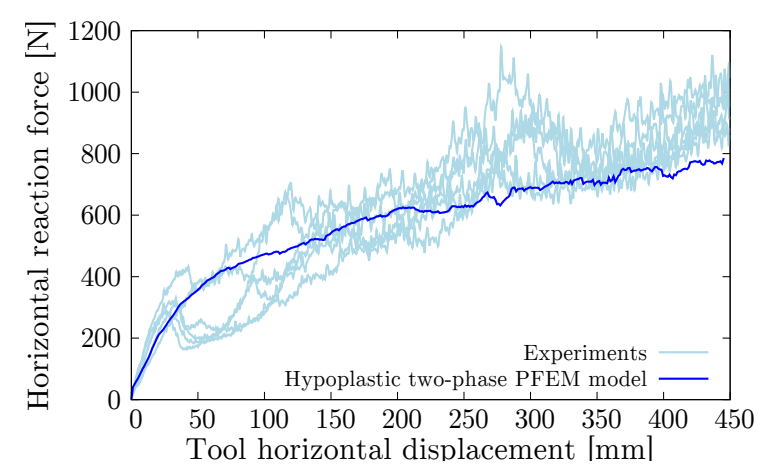

(a) Reference case

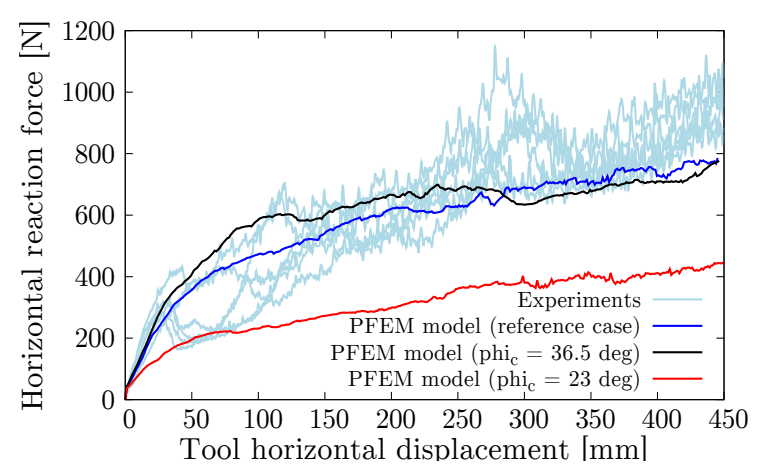

(b) Selected values of $\varphi_{c}$

Figure 3: Evolution of the reaction forces on the cutting tool vs. horizontal tool displacement considering different values for the critical internal friction angle: a) $\varphi_{c}=33^{\circ}$ (reference case) and b) $\varphi_{c}=23^{\circ}, 33^{\circ}$ and $36.5^{\circ}$. (light blue lines: Experiments. Blue, red and black lines: Hypoplastic two-phase PFEM model).

\subsection{Influence of the critical friction angle of the soil $\left(\varphi_{c}\right)$}

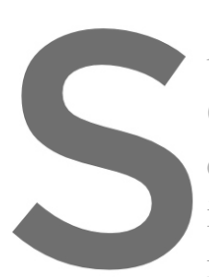

\section{The influence of the}

two results from num

Comparing the topolog

continuous free surface

increased to $36,5^{\circ}$, two

results, it is apparent that a higher critical
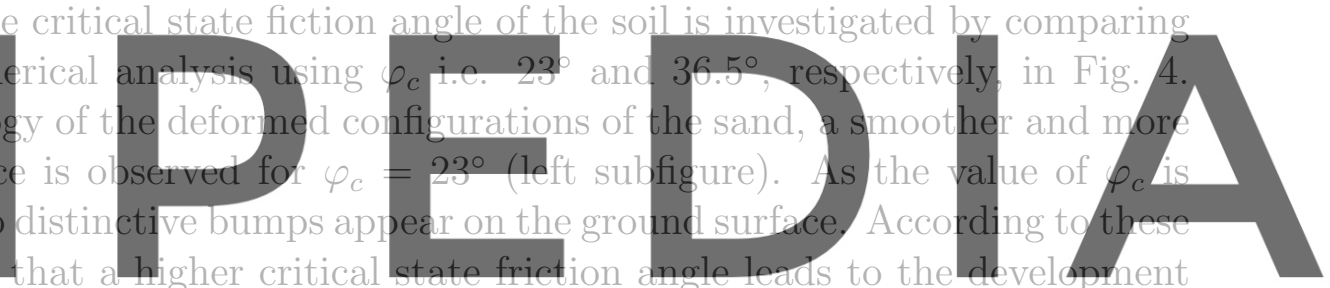

of sharp discontinuities in the ground associated with the sequential activation of shear

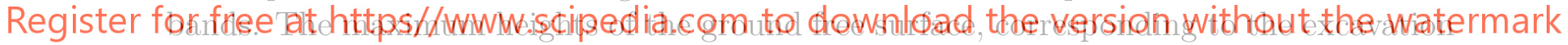

simulations performed with $\varphi_{c}=23^{\circ}$ and $36.5^{\circ}$ are $\approx 202 \mathrm{~mm}$ and $196 \mathrm{~mm}$, respectively.

The infuence of $\varphi_{c}$ in the evolution of the tool reaction forces, is examined in Fig. 3b). It shows, that higher critical friction angles of the soil also lead to higher reaction forces in the tool. For the excavation simulation performed with $\varphi_{c}=23^{\circ}$, the computed maximum reaction force is around $403 \mathrm{~N}$, whereas for the reference case $\left(\varphi_{c}=33^{\circ}\right)$ it is $786 \mathrm{~N}$. Simulations with $\varphi_{c}=36.5^{\circ}$ show higher reaction forces as compared to the reference case during the first $280 \mathrm{~mm}$ of tool horizontal displacement.

\section{SUMMARY AND CONCLUSSIONS}

In this contribution, a two-phase PFEM formulation in conjunction with a hypoplastic constitutive model was presented for the analysis of tool-soil interactions in partially saturated soils. For the validation of the model, excavation experiments have been performed in a box filled with initially fully saturated sand. The proposed model has demonstrated good capabilities concerning the prediction of the complex evolution of large deformations of the ground and the evolution of the topology of the ground surface during the 


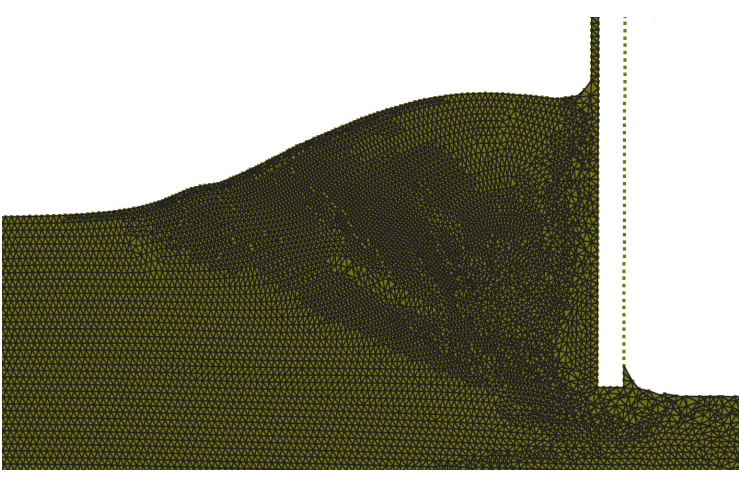

(a) Experiments

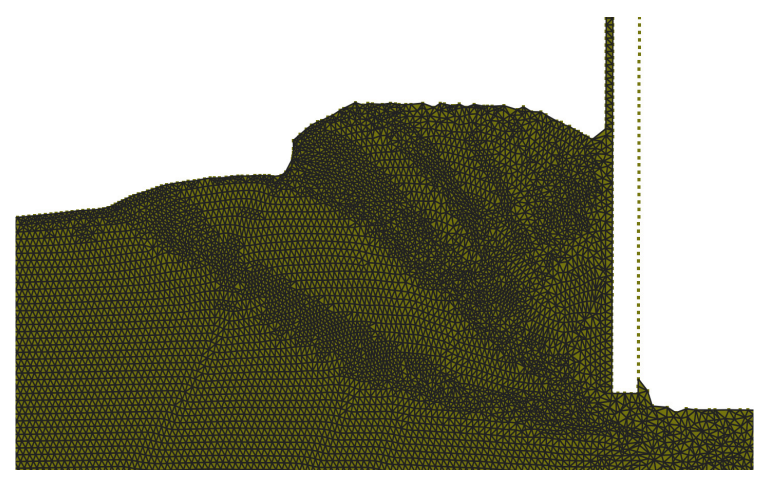

(b) Numerical simulations

Figure 4: Influence of $\varphi_{c}$ on the topology of the deformed soil at a horizontal tool displacement of $S_{x}=300 \mathrm{~mm}$ for two different assumptions for the critical friction angle of the soil. a) $23^{\circ}$ and b) $36.5^{\circ}$.

excavation. Adopting an adaptive mesh refinement scheme controlled at each time step by the relative increase of the void ratio, it was shown, that the model is able to replicate the sequential appearance of shear bands in the soil. Also, the computed reaction forces generated on the tool have shown a good quantitative agreement with the measured forces. It was found however, that the computed forces do not show the oscillatory pattern observed in the measured reaction force-displacement curve. This issue might be connected to intrinsic limitations of the hypoplastic formulation to fully capture the water pore pressure development. On the other hand, it is also expected that a more accurate characterization of the hypoplastic and hydraulic parameters of the soil may yield improved results. Finally, the influence of the critical friction angle of the material was elucidated. Numerical experiments have shown, that a lower critical friction angle leads to a smoother excavation profile, less pronounced shear bands in the ground and smaller tool reaction forces as compared to the reference analysis using calibrated soil parameters.

\section{ACKNOWLEDGEMNET}

Financial support was provided by the German Science Foundation (DFG) in the framework of Subproject C4 of the Collaborative Research Center SFB 837. This support is gratefully acknowledged. The laboratory excavation test device has been developed by Dr. Ulrich Hoppe, and the excavation tests have been performed by Dr. Hoppe, supported by Prof. Dr. Klaus Hackl at Ruhr University Bochum. The authors would like to thank Dr. Hoppe and Prof. Hackl for their continuous collaboration and support during the joint research in Subproject C4 of the SFB 837. The authors also would like to thank Prof. Thorsten Wichtmann (Ruhr University Bochum) for the fruitful discussions on hypoplastic constitutive modeling. 


\section{REFERENCES}

[1] Oñate, E., Idelsohn, S.R., Del Pin, F. and Aubry, R. The particle finite element method. An overview. Int. J. Comput. Methods. (2004) 1:267-307.

[2] Nagel, F. and Meschke, G. An elasto-plastic three phase model for partially saturated soil for the finite element simulation of compressed air support in tunnelling. Int. J. Numer. Anal. Methods Geomech. (2010) 34:605-625.

[3] Truty A. and Zimmermann T. Stabilized mixed finite element formulations for materially nonlinear partially saturated two-phase media. Comput. Methods Appl. Mech. Eng. (2006) 195:1517-1546.

[4] Wood, W.L., Bossak, M. and Zienkiewicz, O.C. An alpha modification of Newmark's method. Int. J. Numer. Methods Eng. (1980) 15:1562-1566.

[5] Calvo, N., Idelsohn, S.R. and Oñate, E. The extended Delaunay tessellation. Eng. Comput. (2003) 20:583-600.

[6] Carbonell, J.M., Oñate, E. and Suárez, B. Modeling of ground excavation with the particle finite-element method. J. Eng. Mech. (2010) 136:455-463.

[7] Van Genuchten, M. Th. A closed-form equation for predicting the hydraulic conductivity of unsaturated soils. Soil Sci. Soc. Am. J. (1980) 44:892-898.

[8] Chapuis, R.P. and Aubertin, M. Predicting the coefficient of permeability of soils using the Kozeny-Carman equation. (Report No. EPM-RT-2003-03) École polytechnique de Montréal (2003).

[9] Von Wolffersdorff, P.A. A hypoplastic relation for granular materials with a predefined limit state surface. Mech. cohesive-frictional mater. (1996) 1:251-271.

[10] Bal, A.R.L., Hoppe, U., Dang, T.S., Hackl, K. and Meschke, G. Hypoplastic particle finite element model for cutting tool-soil interaction simulations: Numerical analysis and experimental validation. Undergr. space (2018). 3:61-71.

[11] Fall, A., Weber, B., Pakpour, M., Lenoir, N., Shahidzadeh, N., Fiscina, J., Wagner, C. and Bonn, D. Sliding friction on wet and dry sand. P.R.L. (2014). 112

[12] Wichtmann, T. and Triantafyllidis, T. An experimental database for the development, calibration and verification of constitutive models for sand with focus to cyclic loading: part I - tests with monotonic loading and stress cycles. Acta Geotech. (2016) 11:739-761.

[13] Aubram, D. An arbitrary Lagrangian-Eulerian method for penetration into sand at finite deformation (PhD thesis). Technische Universität Berlin (2014). 
[14] Jaky, J. Pressure in Silos. Proc. 2nd Int. Conf. Soil Mech. 1:103-107.

[15] Leon Bal, A. and Meschke, G. Two phase Particle Finite Element model for the coupled analysis of cutting tool-soil interaction in partially saturated soft soils. (Submitted) 